\title{
TEACHING AND POPULARIZING ASTRONOMY AND SPACE SCIENCES AT THE OBSERVATORY OF THE CITY OF BOCHUM
}

\author{
J.V. Feitzinger, M. Hünerbein, R. Kordecki, U. Lemmer, G. Monstadt, J. Prölß, \\ Observatory of the City of Bochum, Postfach 102148, D-4630 Bochum 1, F.R. \\ Germany
}

The tasks and aims of the Bochum Observatory are popularization of astronomy and space sciences, and adult education. In general, as a cultural center for natural sciences we must translate scientific nomenclature into the language of the nonspecialist. Astronomy is ideal for presenting the basic facts of scientific methodology and reasoning to the public.

A planetarium is the most versatile instrument for teaching basic astronomy and space sciences to the general public as well as to school groups. We take great care to avoid a lecture-like style in the programs. Audience surveys have shown that most visitors don't want to get the feeling of being educated as in school. Nevertheless, we first have to motivate before we can educate. Instead, most visitors want merely to enjoy astronomy in the pleasant atmosphere of the dome. Consequently, our public planetarium shows contain elements of entertainment. We use many special effects, panoramas, and all-sky projections for a most precise simulation of astronomical phenomena. The audience should get the thrilling impression of witnessing things from close up. For example, they all become passengers on an imaginary spacecraft visiting the rugged terrain of Valles Marineris on Mars, the swirling clouds in Jupiter's atmosphere, or even the vicinity of a whirlpool-like accretion disc around a supermassive black hole in the core of an active galaxy. We use the potential of the planetarium as an "illusion factory" to increase the visitors' positive attitude towards astronomy and space travel. Special music, sound, and noise effects add to the impression.

In addition to astronomical presentations for the general public, we offer special programs devoted to more advanced audiences, such as amateur astronomers. Professional astrophysicists talk about their fields of research and the latest findings in astronomy and related sciences. We also have courses and lectures outside the planetarium dome for a thorough introduction to astronomy.

We should also mention that the planetarium is considered to be a cultural facility in the city of Bochum. Each year we have a couple of exhibitions with painting featuring all aspects of space art. And we present music in the dome together with planetarium projections. These light shows, entitled "Music-Startheater" have run regularly since 1984 with a tremendous impact on our visitor attendance. The three astronomical observing stations have telescopes of $12.5-\mathrm{cm}, 32-\mathrm{cm}, 40-\mathrm{cm}$, and $60-\mathrm{cm}$ diameter.

To ensure a continuing high standard of the performances, the public sky shows in the Bochum Planetarium (20-m dome, 300 seats, Zeiss IV Projector) are 
taped and pre-programmed. Our automation system meets all industrial standards and allows the application of many special effects. Fig. 1 gives a gross overview of its configuration. Its most prominent characteristic is the enormous flexibility. The decentralized architecture allows a modular growth or easy re-design controlled with this automation system.

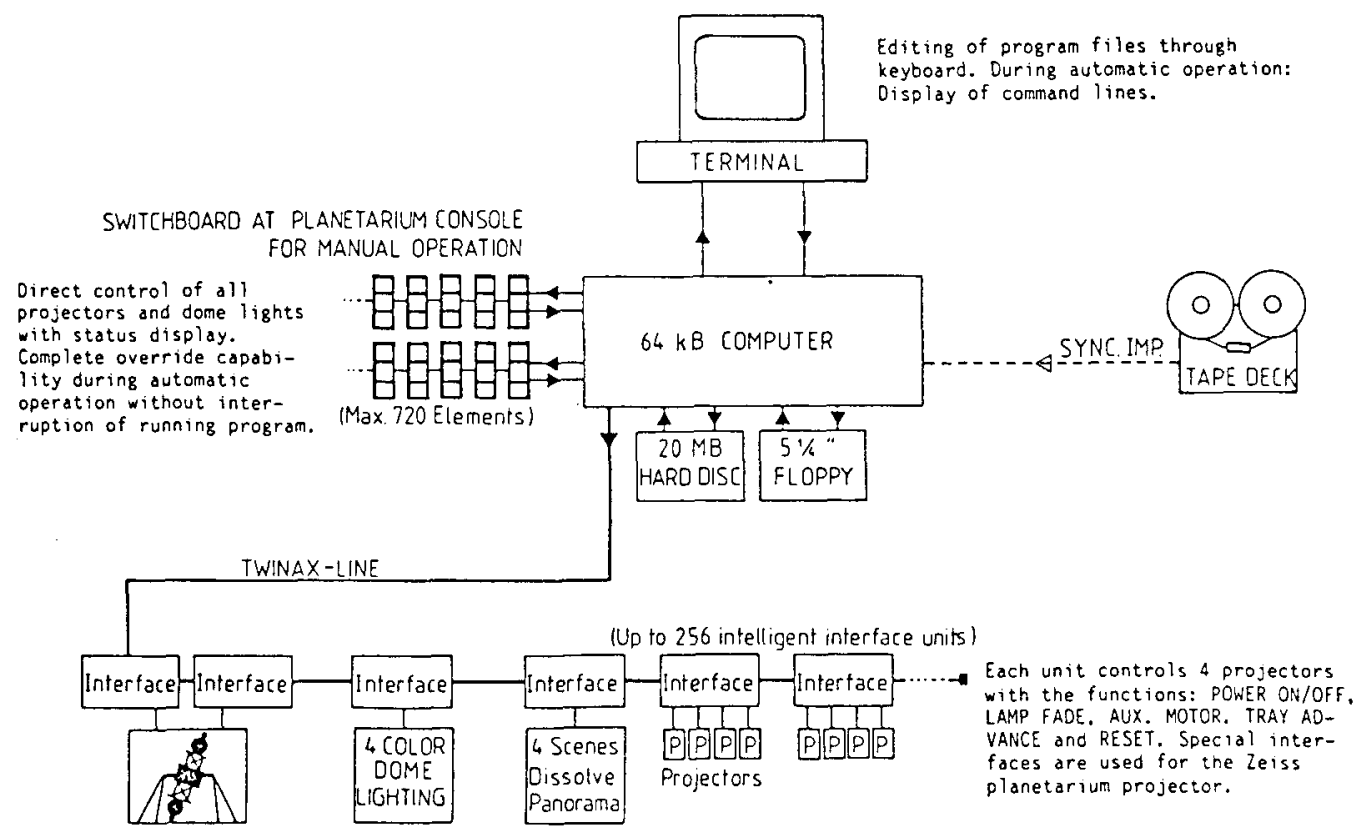

Fig. 1 The Bochum Planetarium Automation System 